\title{
iSPECT Robot - Intelligent Shortest Path and Evading Collision Traveler Robot
}

\author{
M. Varun Kumar \\ Assistant Professor (Junior) \\ School of Information Technology \\ and Engineering \\ VIT University
}

\author{
P.Balamurugavel \\ B.Tech(E.C.E) \\ M.Kumarasamy College of \\ Engineering, Karur ,Tamil Nadu
}

\author{
S.Ganapathi \\ M.S(Software Engineering) \\ School of Information Technology \\ and Engineering \\ VIT University
}

\begin{abstract}
In this paper a minimalist approach to establish obstacle avoidance and course stabilization behavior of an autonomous robot in a region evaluating $2 \mathrm{D}$ virtual world is proposed. The robot uses ultrasonic sensor to evade the hindrances and with distance measurements of barriers predict itself the shortest path to travel on through the destination and updating the virtual environment in database. The robot will be communicated with full duplex communication using ZigBee technology with computer interface. The environment around the rover will be visualized in GUI itself. Even robot can be made navigated searching for maximum light intensity in environment. The proposal is intense to aid the defense system and space exploration. It brings sophisticated method of practice by learning through virtual world of environment which can be enhanced even to 3D with technologies available.
\end{abstract}

\section{General Terms}

- GUI - Graphical User Interface

- ZB1 - ZigBee at Transmitter Section

- ZB2 - ZigBee at Receiver Section

- Dataset table

It is a two dimensional array of size $20 \times 20$ used to store data about the environment to represent virtual 2D world on GUI.

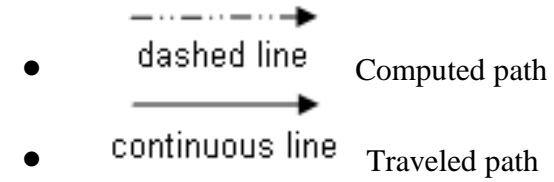

Keywords

- Key terms in dataset $\mathrm{S}-$ Start

E - End

B1, B2, B3, B4 - Blocks

- Key terms for Wheel notation in rover $\mathrm{W}_{\mathrm{rf}}-$ Wheel at front right end

$\mathrm{W}_{\mathrm{lf}}-$ Wheel at front left end

$\mathrm{W}_{\mathrm{rb}}$ - Wheel at back right end

$\mathrm{W}_{\mathrm{lb}}$ - Wheel at back left end

\section{INTRODUCTION}

The purpose of the proposed system is to bring out the innovation in remote navigation system. It endeavors to reach the betterment in the field of defense system and space exploration too. The conventional practice is performed with signal processing techniques for remote sensing of environment. It almost prefers the signals like acoustics or video. The present practice uses navigation through limited automation. We need to command or interpret the robot all through course of its action. This proposal utilizes artificial intelligence for the automation. The proposed system can be made to work with the data studied from the environment on further which is not possible in current trend.

\section{DEPICTION}

The project is a design of a robot with GUI on a personal computer to monitor its functions and operations. The robot will find its start and end position on GUI in application designed using software. It has to travel between them through available shortest path evading the hindrances. The robotic movements are controlled by itself with turns through angle and rotations to take alternate paths in case of barriers on its way. The light seek in the environment can also be performed by the navigator. On increased performance, the robot can be made to travel through multiple nodes with shortest path navigation and collision avoidance. The current system uses signal processing techniques to study the environment with robot and these information's are very difficult for further process. This proposal would resolve this shortcoming and the information about the particular environment will be depicted as virtual world in application software interface. These information would be enough in predicting the shortest path to a particular node before reaching closer to the hindrances.

\section{BLOCK DIAGRAM}

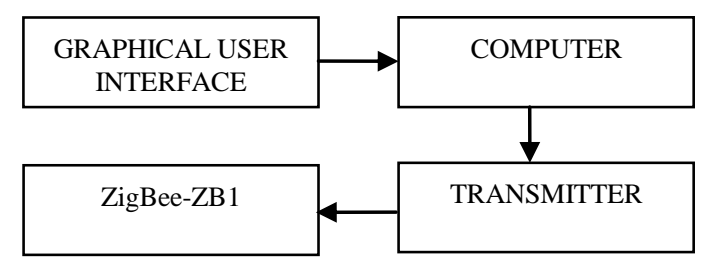

Figure 1: Transmitter Section 


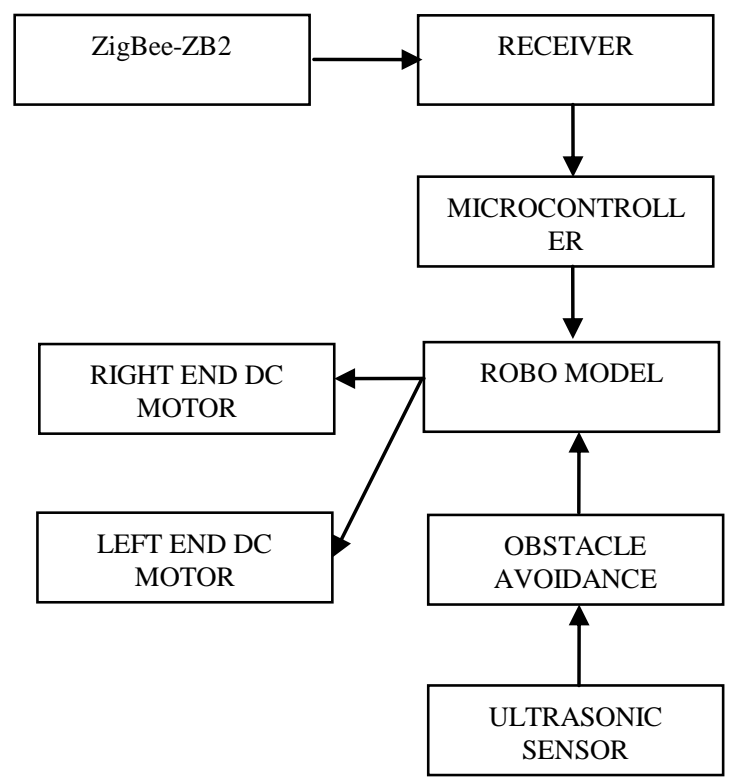

Figure 2: Receiver Section

\section{IMPLEMENTATION TACTIC}

The rover will be commanded to travel from source $\mathrm{S}$ to destination $\mathrm{E}$ on GUI. With wireless transmission, the rover will be activated with movement operation computed for shortest path between nodes. The rover will do operations on motion and look for hindrances for updating dataset table maintained with GUI. On detection of barriers, the application will compute alternate available shortest path towards E through intermediate nodes virtual destination. Figure 4 gives the Virtual 2D environment which is to be visualized as result in GUI. The prominent hardware device is the usual robot model with movements through all possible directions. The robot consists of two pairs of wheel assembly with DC motors supplied by electrical battery power. When the particular task has been given, it may require taking directional motions and turning rotations also. The directional motions will be made by forward and reverse action of the DC motors for wheel movements.

If the robot needs to take right turn, the right end motors will be activated for reverse motion whereas the left end motors will be made for forward motion. See Figure 3a. The similar action will be performed for turning to left just altering the operations of end motors of the robot. See Figure 3b. These end motion pair operation is performed to keep the centroid position of our robot remain unaltered in the virtual representation.The ultrasonic sensor at the roof of the rover will be capable of rotating for scan the obstacles on its view. On any detection, the delay in time will be used to compute the distance of the hindrance at that particular angle. These particulars will be transmitted to the computer interface application through full duplex ZigBee communication for updating the $2 \mathrm{D}$ virtual environment.

Figure 5 gives the details about all particulars in the environment through dataset table. By default all cells in dataset table will be set to 0 which denotes they are free to travel on. When start $\mathrm{S}$ and end $\mathrm{E}$ has been set on GUI, the corresponding cell in dataset table take the value of 2 and 3 for $\mathrm{S}$ and $\mathrm{E}$ respectively. When rover detects and hindrance through ultrasonic, the distance of that barrier will be computed and dataset cell will be updated to 1 to denote block. As rover is commanded to travel, application will fetch shortest distance through computation of inclination angle and travel distances. The rover will follow instructions from application and executes itself through angle turns and movements. The updated dataset table when it travels from $\mathrm{S}$ to $\mathrm{E}$ by taking alternate path available through intermediate node which can be represented with integers $3,4,5$, etc. is shown in Figure 6.
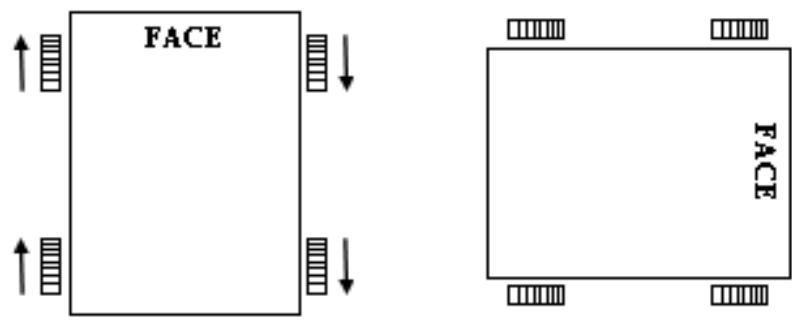

Figure 3: Turning towards right

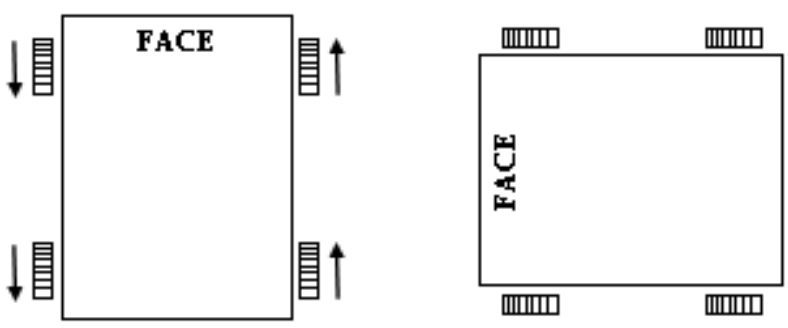

Figure 4: Turning towards left

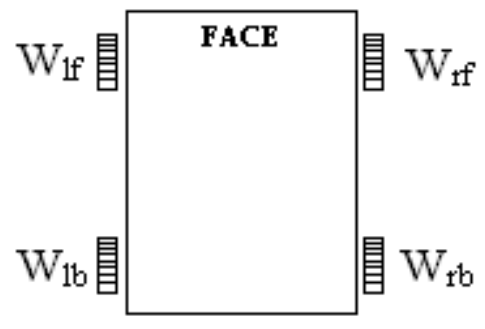

Figure 5: Rover model with wheel labels

\section{COMPUTATION}

The virtual environment dataset table is illustrated in Figure 4. In that, $S$ reads cell $(1,1)$ and $E$ reads cell $(16,16)$. Computer detects the $\mathrm{E}$ is situated at 15 column (x) apart and 15 row (y) ahead from S. With these nodes, the shortest path will be straight line connecting these nodes. The rover has to be rotated about turn angle $\theta$. The $\theta$ will be computed with formula, $\theta=\tan -1(\mathrm{x} / \mathrm{y})$

After rotation about centroid of rover, the rover has to take hypotenuse distance travel hyp which will be hyp $=\mathrm{x} / \cos \theta$ or

hyp $=\mathrm{y} / \sin \theta$ Completing the travel through hypotenuse (hyp) distance, it will again check for rotation through angle depending on availability of further node travel for reach. On travel through this path, ultrasonics reflected from barrier used to denote hindrances in the dataset table. The location of hindrance is taken by halving the distance traveled by ultrasonic from rover at particular angle. The datas of location of hindrance and angle are

transmitted through wireless with ZigBee to application and dataset will get updated. 


\section{INIMITABLE FEATURES}

Autonomous, no interpretations required on rover through its course

Shortest path travel between $\mathrm{S}$ and $\mathrm{E}$ on available route

Evasion of hindrances

Development of Virtual 2D environment

Dynamic dataset to read particular environment

Efficient computation of path with predicting intermediate nodes after developing enough dataset table

$\square \quad$ Full duplex wireless communication between GUI and rover

Table 1: Actions and DC motor operations

\begin{tabular}{|l|l|l|l|l|}
\hline \multicolumn{1}{|c|}{ Actions } & \multicolumn{4}{|c|}{ DC Motor Operations } \\
\hline & \multicolumn{1}{|c|}{$\mathbf{W}_{\mathbf{r f}}$} & \multicolumn{1}{c|}{$\mathbf{W}_{\mathbf{I f}}$} & \multicolumn{1}{c|}{$\mathbf{W}_{\mathbf{r b}}$} & \multicolumn{1}{c|}{$\mathbf{W}_{\mathbf{l b}}$} \\
\hline Forward & forward & forward & forward & forward \\
\hline Right turn & reverse & forward & reverse & forward \\
\hline Left turn & forward & reverse & forward & reverse \\
\hline Backward & reverse & reverse & reverse & reverse \\
\hline
\end{tabular}

\section{STAND-IN SOLUTIONS}

The conventional practice uses rovers for navigation through semi automation. They will be automating the robot through remote operation and they have to take measures through manual

processing of data from the environment like heat, obstacles, light and other parameters.
PROS

Efficient intelligent computation of

shortest path

Obstacle avoidance

ZigBee full duplex communication

Wireless activation and control of rover

Online access of remote environment

\subsection{ALTERCATIONS}

The substitute for this proposal will be tedious as practicing the conventional method. We could go for signal processing techniques like digital image processing or video processing. This practice will require lot of memory spaces and much complex algorithms and development to work with the streaming video data and multiple images and its comparison.

\section{RESOURCES}

The reserves that are required for this proposal is a computer system with any of Windows platform installed for support of developed GUI application. The computer should capable of communicating with the rover through port devices for access through the transmitter kit with ZigBee ZB1. The receiver also takes the wireless information through ZigBee ZB2 to activate programmed microcontroller for operating the robot. The GUI platform and all computations will be processed and developed in software environment.

\section{ILLUSTRATION}

The illustration taken for navigation of 4 sq.mt area alone which is $2 \mathrm{~m}$ wide and $2 \mathrm{~m}$ length, a square region. The distance units are taken in centimeters and the virtual world is represented from 20x20 two dimensional matrix array.

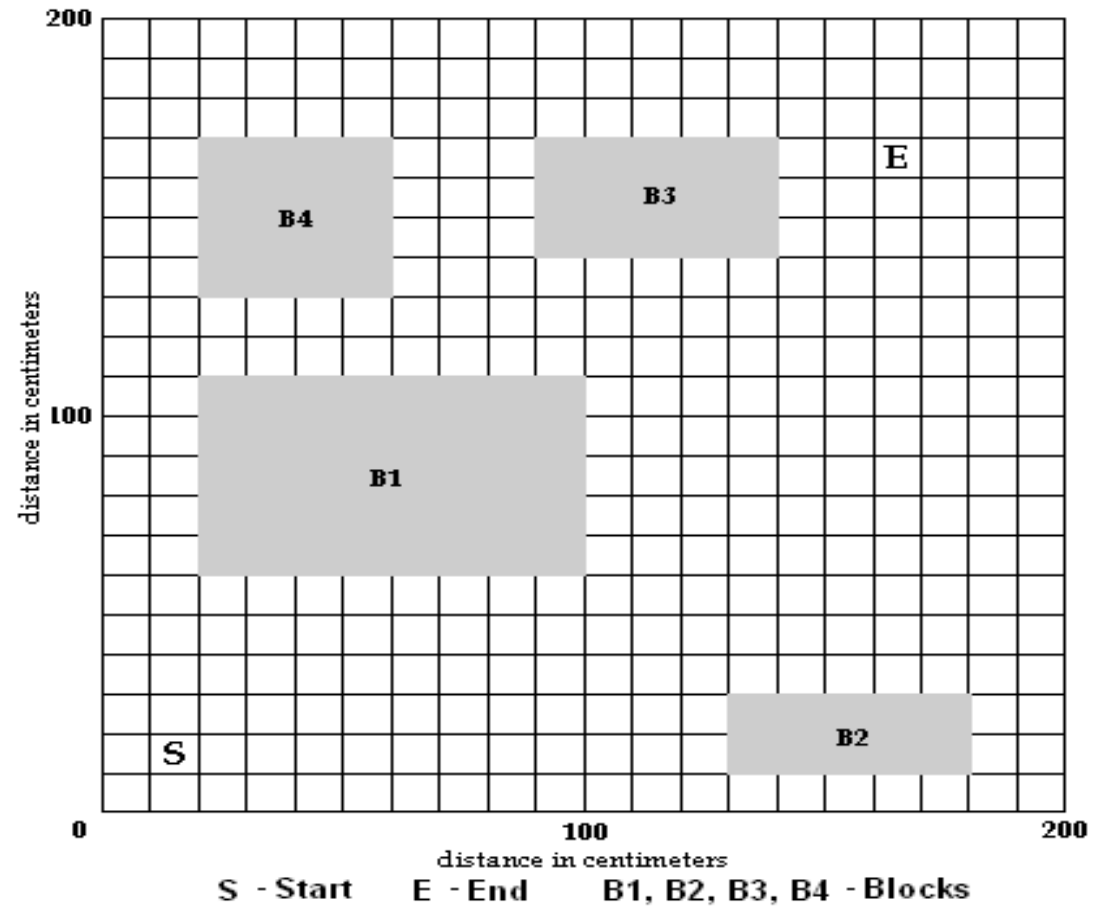

Figure 6: Virtual 2D Environment 


\begin{tabular}{|c|c|c|c|c|c|c|c|c|c|c|c|c|c|c|c|c|c|c|c|}
\hline $\mathbf{0}$ & 0 & 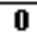 & 0 & 0 & 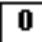 & U & 0 & 0 & 0 & 0 & 0 & 0 & 0 & 0 & 0 & 0 & $\mathbf{0}$ & 0 & 0 \\
\hline 0 & 0 & 0 & $\mathbf{0}$ & 0 & 0 & $\mathbf{0}$ & 0 & 0 & $\mathbf{0}$ & 0 & 0 & 0 & 0 & 0 & $\mathbf{0}$ & 0 & 0 & 0 & $\mathbf{0}$ \\
\hline 0 & $\mathbf{0}$ & $\mathbf{0}$ & $\mathbf{0}$ & n & 0 & $\mathbf{0}$ & $\mathbf{n}$ & n & $\mathbf{0}$ & $\mathbf{0}$ & n & 0 & \begin{tabular}{|l|l|}
$\mathbf{0}$ \\
\end{tabular} & n & 0 & $\mathbf{0}$ & $\mathbf{0}$ & $\mathbf{0}$ & 0 \\
\hline 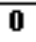 & $\mathbf{0}$ & 1 & 2 & 1 & 1 & 0 & 0 & 0 & $\mathbf{2}$ & 1 & $T$ & 1 & $\mathbf{1}$ & 0 & 0 & 3 & 0 & 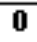 & 0 \\
\hline$\overline{\mathbf{0}}$ & 0 & 1 & \begin{tabular}{|l|l}
0 \\
\end{tabular} & 0 & 1 & 0 & 0 & 0 & 1 & 0 & $\mathbf{0}$ & 0 & $\mathbf{1}$ & $\mathbf{0}$ & 0 & 0 & $\overline{0}$ & $\mathbf{0}$ & $\mathbf{0}$ \\
\hline 0 & $\mathbf{0}$ & 1 & 0 & 0 & $\mathbf{1}$ & $\mathbf{0}$ & 0 & 0 & 1 & 1 & 1 & 1 & 1 & 0 & 0 & 0 & 0 & 0 & 0 \\
\hline 0 & 0 & 1 & 1 & 7 & & $\mathbf{0}$ & 0 & & $\mathbf{0}$ & n & & $\mathrm{n}$ & $\mathbf{0}$ & 0 & n & $\mathbf{0}$ & $\mathbf{0}$ & $\mathbf{0}$ & 0 \\
\hline 0 & $\overline{0}$ & $\overline{0}$ & $\mathbf{0}$ & 0 & 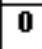 & 0 & 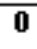 & 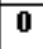 & $\mathbf{0}$ & 0 & 0 & $\mathbf{0}$ & $\mathbf{0}$ & 0 & 0 & 0 & $\mathbf{0}$ & $\mathbf{0}$ & 0 \\
\hline $\mathbf{0}$ & $\mathbf{0}$ & $\mathbf{0}$ & 0 & 0 & 0 & 0 & 0 & $\mathbf{0}$ & $\mathbf{0}$ & 0 & 0 & 0 & $\mathbf{0}$ & 0 & 0 & 0 & $\mathbf{0}$ & 0 & 0 \\
\hline $\mathbf{0}$ & 0 & $\mathbf{1}$ & 1 & 1 & $\mathbf{l}$ & 1 & $\mathbf{1}$ & 1 & 1 & n & n & 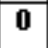 & \begin{tabular}{|l|l|} 
\\
\end{tabular} & n & n & 0 & 0 & $\mathbf{0}$ & $\mathbf{0}$ \\
\hline $\mathbf{0}$ & 0 & 1 & $\mathbf{0}$ & 0 & 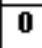 & $\mathbf{0}$ & 8 & & 1 & 0 & & $\mathbf{0}$ & 0 & 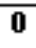 & 0 & 0 & 0 & $\mathbf{0}$ & $\mathbf{0}$ \\
\hline $\mathbf{0}$ & I & 1 & 0 & 0 & 0 & 0 & 0 & 0 & 1 & $\mathbf{U}$ & $\mathbf{0}$ & \begin{tabular}{|l|}
0 \\
\end{tabular} & \begin{tabular}{|l|l|}
0 \\
\end{tabular} & $\mathbf{0}$ & $\mathbf{0}$ & $\mathbf{0}$ & 0 & 0 & $\overline{\mathbf{0}}$ \\
\hline$\overline{\mathbf{0}}$ & $\overline{\mathbf{0}}$ & 1 & 0 & $\overline{0}$ & 0 & 0 & $\mathbf{0}$ & 0 & 1 & $\mathbf{0}$ & $\overline{0}$ & 0 & \begin{tabular}{|l|}
0 \\
\end{tabular} & $\overline{0}$ & 0 & 0 & 0 & $\mathbf{0}$ & $\overline{0}$ \\
\hline $\mathbf{0}$ & $\overline{\mathbf{0}}$ & 1 & 1 & 1 & 1 & 1 & 1 & 1 & 1 & 0 & $\mathbf{0}$ & n & $\mathbf{0}$ & $\mathbf{0}$ & $\mathbf{0}$ & 0 & 0 & $\mathbf{0}$ & $\overline{0}$ \\
\hline $\mathbf{0}$ & 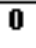 & $\mathbf{0}$ & 0 & $\mathbf{0}$ & $\mathbf{0}$ & 0 & 0 & $\mathbf{0}$ & $\mathbf{0}$ & 0 & 0 & 0 & $\mathbf{0}$ & 0 & $\mathbf{0}$ & $\mathbf{0}$ & 0 & 0 & $\overline{0}$ \\
\hline $\mathbf{0}$ & 0 & 0 & 0 & 0 & 0 & 0 & 0 & \begin{tabular}{|l}
$\mathbf{0}$ \\
\end{tabular} & $\mathbf{0}$ & $\mathbf{0}$ & 4 & 0 & $\mathbf{0}$ & $\mathbf{0}$ & $\mathbf{0}$ & 0 & 0 & $\mathbf{0}$ & 0 \\
\hline $\mathbf{0}$ & 0 & $\mathbf{0}$ & 0 & $\mathbf{0}$ & $\mathbf{0}$ & 0 & $\mathbf{0}$ & n & 0 & $\mathbf{0}$ & $\mathbf{0}$ & 0 & $\mathbf{0}$ & 0 & 8 & 0 & $\mathbf{0}$ & $\mathbf{0}$ & 0 \\
\hline $\mathbf{0}$ & $\overline{0}$ & $\mathbf{0}$ & 0 & $\mathbf{0}$ & 0 & 0 & $\mathbf{0}$ & 0 & 0 & 0 & $\mathbf{0}$ & $\mathbf{0}$ & 1 & $\mathbf{1}$ & 1 & $\mathbf{1}$ & $\mathbf{1}$ & $\mathbf{0}$ & 0 \\
\hline $\mathbf{0}$ & 2 & $\mathbf{0}$ & 0 & 0 & 0 & 0 & 0 & 0 & 0 & 0 & 0 & 0 & $\mathbf{1}$ & 1 & $\mathbf{1}$ & 1 & 1 & 0 & 0 \\
\hline$\overline{0}$ & 0 & 0 & 0 & $\mathbf{0}$ & 0 & 0 & 0 & 0 & 0 & 0 & 0 & 0 & $\mathbf{0}$ & $\mathbf{0}$ & $\mathbf{0}$ & $\mathbf{0}$ & 0 & $\mathbf{0}$ & 0 \\
\hline
\end{tabular}

Figure 7: Final dataset after complete exploration of environment

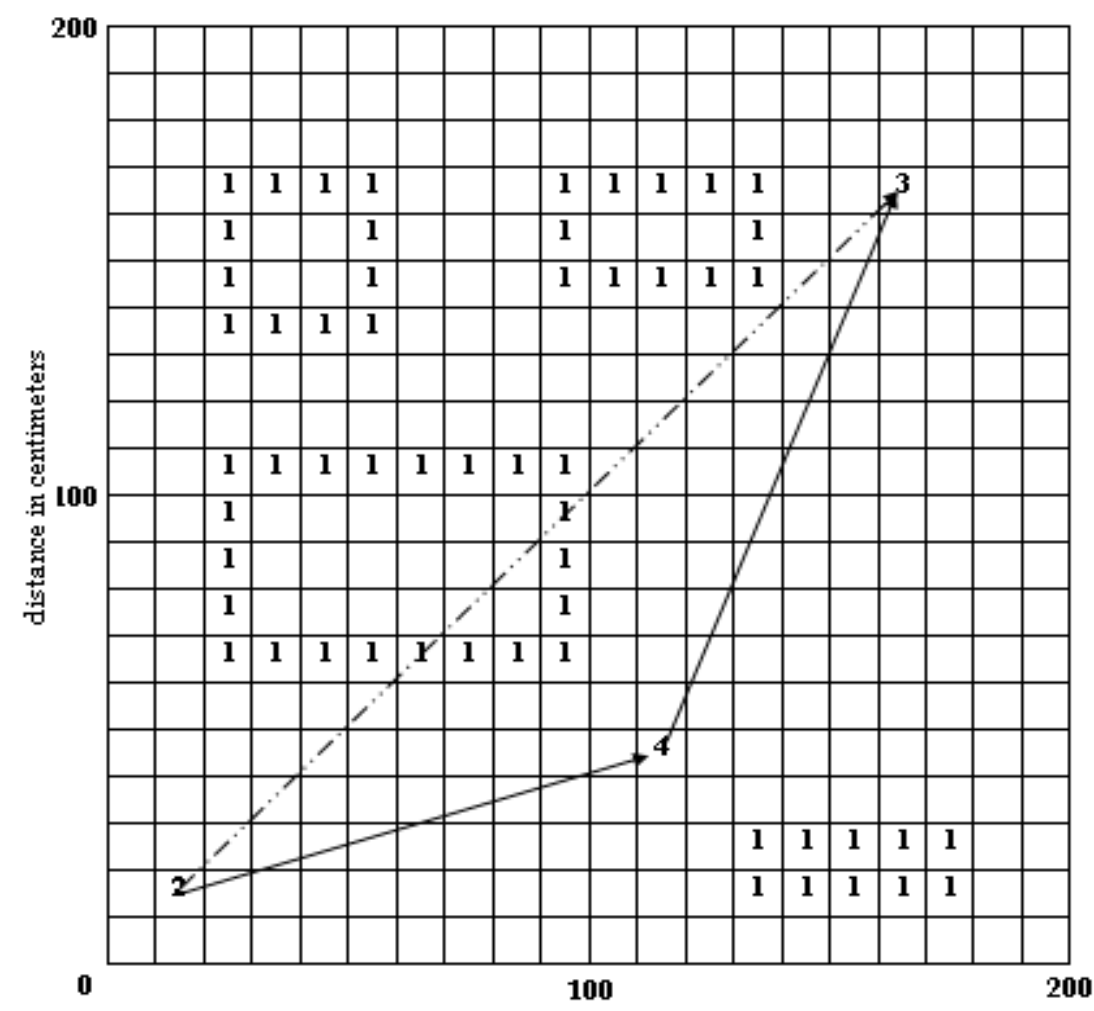

distance in centimeters

Figure 8: Data table representing virtual environment with complete navigation ( 0 has been intentionally left blank) 


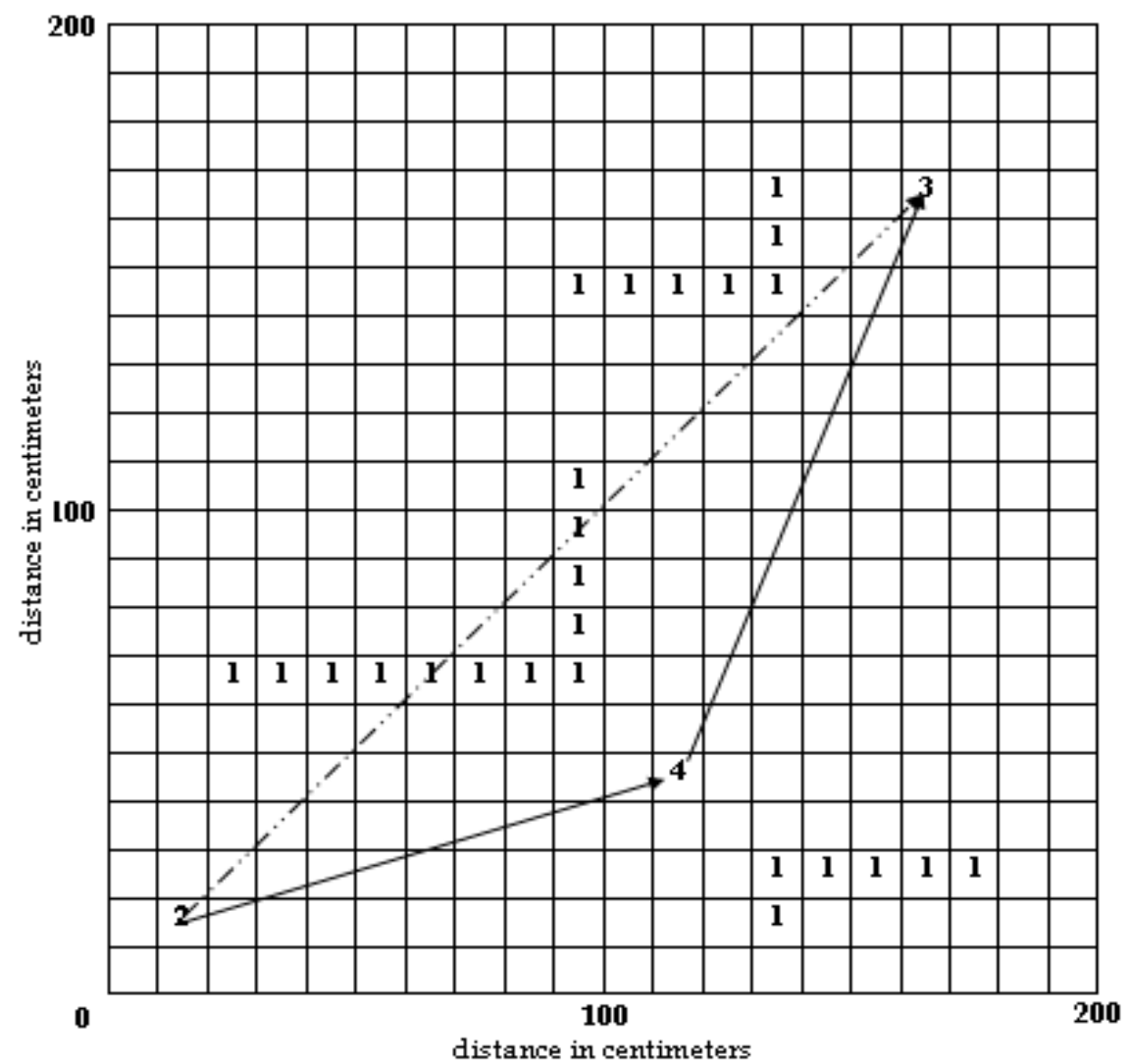

Figure 9: Data table while rover taking to $\mathrm{E}$ from $\mathrm{S}$

Data values: 0 - Free space (In Illustration, it has been intentionally left blank)

$$
\begin{aligned}
& \text { 1- Detected hindrances } \\
& 2 \text { - Start position } \\
& 3 \text { - End position } \\
& 4 \text { - Intermediate travel node }
\end{aligned}
$$

\section{REFERENCES}

[1] www.gamasutra.com

[2] www.policyalmanac.org/games/aStarTutorial.html

[3] www.red3d.com/cwr/steer

[4] PDF : The Long and Short of Steering in Computer Games www.ducati.doc.ntu.ac.uk/uksim/uksim'04/Papers/Simon\% 20Tomlinson-\%2004-20/paper04-20\%20CR.pdf

[5] Microcontroller Based Infra-red Tracking

[6] Robot-EFY April 2007

[7] PC Based Mobile Robot For Navigation-EFY October 2008
[8] "Superhuman Performance of Surgical Tasks by Robots Using Iterative Learning from Human-Guided Demonstrations" by Jur van den Berg, Daniel Duckworth, Stephen Miller, Humphrey Hu, Andrew Wan, Xiao-Yu Fu, Ken Goldberg and Pieter Abbeel.

[9] "Tools and Algorithms for Mobile Robot Navigation with Uncertain Localization" Ph.D. Thesis by Kristopher Kriechbaum. Mechanical Engineering June, 2006.

[10] "Algorithms for Mobile Robot Localization and Mapping, Incorporating Detailed Noise Modeling and Multi-Scale Feature Extraction" Ph.D. Thesis by Samuel Pfister. Mechanical Engineering June, 2006.

[11] "A Robot that Walks; Emergent Behaviors from a Carefully Evolved Network"- Rodney A. Brooks (MIT Artificial Intelligence Laboratory, 545 Technology Square, Cambridge,
02139 , 